\title{
IMAGE DENOISING USING BILATERAL FILTER IN HIGH DIMENSIONAL PATCH-SPACE
}

\author{
Quoc Bao DO, Azeddine BEGHDADI, Marie LUONG
}

\author{
L2TI, Université Paris 13, France
}

\begin{abstract}
This paper proposes a new noise filtering method inspired by Bilateral filter (BF) and non-local means (NLM) filter. The main idea here is to perform the $\mathrm{BF}$ in a multidimensional patch-space using an anisotropic kernel. The filtered multidimensional signal is then transformed back onto the image spatial domain to yield the desired enhanced image. The proposed method is compared to the state-of-art. The obtained results are highly promising.
\end{abstract}

Index Terms-Denoising, Bilateral, Non-local means, high dimensional space.

\section{INTRODUCTION}

Noise filtering is one of the most studied problem in image processing. Many approaches have been then proposed in the literature. The filtering process could be performed in the image space domain or in the transformed domain. In spatial domain methods the pixel intensity is replaced by an average value computed in a neighborhood in order to smooth the signal irregularities and especially such noise. However, some salient features such as edges and useful details may be affected by the smoothing effect. To overcome this drawback some approaches have been proposed to adapt the filtering strength to the local content of the signal according to some objective criteria related to a given model. These methods are based on the observation that any image often contains self-similarity and some spatial redundancy that could be exploited in the design of the filter. Indeed, if the noise is considered as an independent and identically distributed (i.i.d.) random signal, it could be smoothed out by averaging similar pixels. In [1], the authors propose Bilateral Filter (BF) which takes into account both spatial and intensity information to define similar pixels for a given one. The similarities between BF and anisotropic filtering have been inversigated in [2-4]. Another adaptive filtering approach, called non-local means (NLM) [5-7], has been recently proposed. Instead of using pixel-based similarity as in BF, NLM proposes to use patchbased similarity which makes the method more robust in textured and contrasted regions. Many methods for improving the performance of NLM have been proposed. In [8] an iterative NLM has been proposed. The computational time is also reduced in [9]. NLM in the wavelet domain is presented in [10]. In [11], authors propose a transform which maps each patch in the image domain to a point in a high dimensional space (called patch-space) and show that NLM algorithm could be expressed as an isotropic filter in this new space. In this paper, we propose to use BF, i.e. an anisotropic filter, in the patch-space to improve the denoising performance.

The paper is organized as follows: section 2 is devoted to a short review of BF and NLM, the high dimensional patch-space is presented in section 3. The proposed method is described in section 4 followed by experimental results in section 5 . The conclusions are finally given in section 6 .

\section{RELATED WORKS}

Let us define a 2D noise-free image $u: \Omega \in \mathbb{R}^{2} \rightarrow \mathbb{R}$. Its noisy version $v$ at pixel $(x, y)$ defined as $v(x, y)=u(x, y)+$ $n(x, y)$ where $n$ is identical, independent Gaussian noise. The aim of denoising is to estimate $u$ from $v$. Both BF and NLM restore noisy pixel by averaging the neighboring pixels. An unifying formula for these methods could be expressed as follows:

$$
\hat{u}(x, y)=\frac{\sum_{\left(x^{\prime}, y^{\prime}\right) \in \Omega} w\left(x, y, x^{\prime}, y^{\prime}\right) v\left(x^{\prime}, y^{\prime}\right)}{\sum_{\left(x^{\prime}, y^{\prime}\right) \in \Omega} w\left(x, y, x^{\prime}, y^{\prime}\right)}
$$

where $\Omega$ is the image domain and $w\left(x, y, x^{\prime}, y^{\prime}\right)$ stands for the weight which corresponds to the similarity between pixel $v(x, y)$ and $v\left(x^{\prime}, y^{\prime}\right)$. Indeed, each method proposes a kernel to estimate this weight. According to $\mathrm{BF}[1]$, the kernel is defined as follows:

$w_{B F}\left(x, y, x^{\prime}, y^{\prime}\right)$

$=\exp \left(\frac{-\left(\left\|x-x^{\prime}\right\|^{2}+\left\|y-y^{\prime}\right\|^{2}\right)}{h_{s}^{2}}\right) \exp \left(\frac{-\left\|v(x, y)-v\left(x^{\prime}, y^{\prime}\right)\right\|^{2}}{h_{r}^{2}}\right)$

where $h_{s}$ and $h_{r}$ are space and range parameters, respectively. Note that this filter takes into account both spatial and intensity information. In [2-4], it has been proven that $\mathrm{BF}$ is an anisotropic filter with a special regularization term. In the case of NLM, its weight is given by:

$w_{N L M}\left(x, y, x^{\prime}, y^{\prime}\right)=\exp \left(\frac{-G_{a} *\left\|N(x, y)-\boldsymbol{N}\left(x^{\prime}, y^{\prime}\right)\right\|^{2}}{h_{r}^{2}}\right)_{(3)}$ where $N(x, y), N\left(x^{\prime}, y^{\prime}\right)$ are two small patches of size $r \times r$ around the pixel $(x, y)$ and $\left(x^{\prime}, y^{\prime}\right)$, respectively ( $r$ is usually equal to 7), $G_{a}$ is a Gaussian kernel with standard deviation 
$a$ of the same size as $N(x, y), N\left(x^{\prime}, y^{\prime}\right)$. This weight means that the pixel $\left(x^{\prime}, y^{\prime}\right)$, whose patch $N\left(x^{\prime}, y^{\prime}\right)$ is similar to $N(x, y)$ will have greater impact in the average. Moreover, the patch-similarity measure is weighted by the Gaussian kernel $G_{a}$ to give more weight to pixels close to the patch center. Note that NLM considers only intensity information. Due to computational burden, instead of considering all pixel $\left(x^{\prime}, y^{\prime}\right)$ in the noisy image, Buades et al. propose to restraint a search window $\Omega(\mathrm{x}, \mathrm{y}) \in \Omega$ which is usually set equal to $21 \times 21$, i.e. there are 441 patch candidates. Indeed, further analyses [12] have shown that if the size of $\Omega(\mathrm{x}, \mathrm{y})$ increases, more bias is introduced due to several mismatching patches that are taken into account. In the other work $[13,14]$, the authors used a strategy where only the best candidates are selected by exploring the entire image plane. Counter intuitively, this approach yields worse result in both term of subjective and objective measurement (see Fig.1 - please use your monitor to view all the images in this paper). In flat regions, the noise pattern of a given-patch will match well with that of the best candidates. Averaging these similar noise patterns cannot effectively remove the noise. We refer to this as "best-worse paradox" in the sense that if we consider only the best candidates, the result is worse. Based on these remarks, the semi-non local approach, i.e. restrained to a small window $\Omega(\mathrm{x}, \mathrm{y})$, is used in this work.
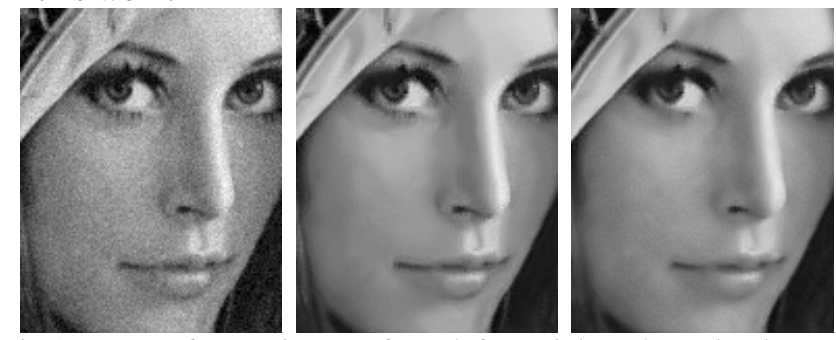

Fig.1: zoom of Lena image (from left to right): the noisy image, the restored image with semi-non local approach, and selective NLM, respectively. Note that the first approach yields better result than the second one.

\section{HIGH DIMENSIONAL PATCH-SPACE}

The NML's weight in equation (3) can be rewritten as follows:

$$
\begin{aligned}
& w_{N L M}\left(x, y, x^{\prime}, y^{\prime}\right) \\
& =\exp \left(\frac{-\left\|\sqrt{G_{a}} N(x, y)-\sqrt{G_{a}} \boldsymbol{N}\left(x^{\prime}, y^{\prime}\right)\right\|^{2}}{h_{r}^{2}}\right)
\end{aligned}
$$

Note that a weighted patch $\sqrt{G_{a}} \boldsymbol{N}(x, y)$ of size $r \times r$ can be expressed as a $r^{2}$-dimensional vector. In the next, we will describe how to map an image patch onto the high dimensional patch-space and how to project pixel values from this new space back onto the image spatial domain.

Mapping in the patch-space:

We define $r^{2}$ dimensional patch-space noted $\Psi \in$ $\mathbb{R}^{r^{2}}$ where the coordinates of each point $\boldsymbol{p}$ in this space are the intensity values of a weighted patch $\sqrt{G_{a}} N$ in the image domain $\Omega$. In other words, the intensity values in the image domain $\Omega$ become spatial coordinates in the new high dimensional patch-space $\Psi$. Each value $\boldsymbol{V}$ of a point $\boldsymbol{p}$ in this space is defined as follows:

$$
\boldsymbol{V}(\boldsymbol{p})=\left(V_{1}(\boldsymbol{p}), V_{2}(\boldsymbol{p})\right)=(v(x, y), 1) \text { if } \boldsymbol{p}=\sqrt{G_{a}} \boldsymbol{N}(x, y)
$$

Note that $\boldsymbol{V}(\boldsymbol{p})$ contains two components:

- The first one $V_{1}(\boldsymbol{p})=v(x, y)$ (the gray level of center pixel $(x, y)$ of the patch $N(x, y))$.

- $\quad$ The second one $V_{2}(\boldsymbol{p})$ is always set equal to 1 .

\section{Back-projection on the image domain:}

Instead of filtering directly the pixel value in the image domain, we alter the multi-values $\boldsymbol{V}(\boldsymbol{p})$ in the patch-space to obtain $\widehat{\boldsymbol{U}}(\boldsymbol{p})$ (the filtering method in this space will be discussed in the next section). This filtered value is then transformed back onto the image domain $\Omega$ as follows:

$$
\hat{u}(x, y)=\frac{\widehat{U}_{\mathbf{1}}(\boldsymbol{p})}{\widehat{U}_{2}(\boldsymbol{p})} \text { if } \boldsymbol{p}=\sqrt{G_{a}} \boldsymbol{N}(x, y) \text { and }(x, y) \in \Omega
$$

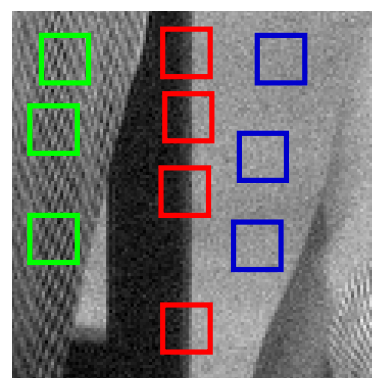

Image patch in the image space

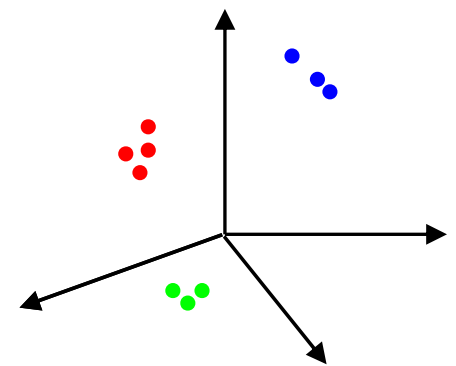

Corresponding points in the high dimensional patch-space
Fig. 2: An illustration of mapping image patch onto high dimensional patch-space. Each patch is presented by a corresponding point in the new space.

Note that the patch-space is a sparse one where only points corresponding to the patches in the image domain are defined.

\section{PROPOSED METHOD}

To restore the pixel $(x, y)$, in order to avoid "best-worse paradox" phenomenon, instead of projecting all patches in the image domain $\Omega$ onto the patch-space $\Psi$, we project only the patches on the sub-domain $\Omega(\mathrm{x}, \mathrm{y})$ (small windows of size $21 \times 21$ around the current pixel $(x, y)$ ) and carry out the filtering on these projected values. Since all values in weighted patch $\sqrt{G_{a}} \boldsymbol{N}(x, y)$ become spatial coordinates $\boldsymbol{p}$ in the patch-space, we can rewrite equation (1) of NLM as follows:

$$
\widehat{u}(x, y)=\frac{\widehat{U}_{1}(\boldsymbol{p})}{\widehat{U}_{2}(\boldsymbol{p})}=\frac{\sum_{\boldsymbol{q} \in \Psi} \exp \left(\frac{-\|\boldsymbol{p}-\boldsymbol{q}\|^{2}}{h_{r}^{2}}\right) V_{1}(\boldsymbol{q})}{\sum_{\boldsymbol{q} \in \Psi} \exp \left(\frac{-\|\boldsymbol{p}-\boldsymbol{q}\|^{2}}{h_{r}^{2}}\right) V_{2}(\boldsymbol{q})}
$$

Note that both nominator and denominator in this equation can be interpreted as Gaussian filter in the patch- 
space. Therefore, we can summarize NLM in the two following steps:

- Step 1: Gaussian filtering in the patch-space

- Step 2: Projection back onto the image space by using the division of two components of the filtered values (equation (5))

It is worth to notice that the Gaussian filter in the first step is an isotropic filter. Here, we propose to replace it by an anisotropic filter in order to make it more adapted to local structure. In the literature, there are many anisotropic diffusion methods such as Total Variation[15], PeronaMalik's method[16] which mimic physical processes by locally diffusing pixel values along the image structure. Since these methods are local based, their adaptation to a such sparse patch-space is rather a difficult task. However, as discussed above, BF acts as an anisotropic filter and it works in non-local manner therefore it could be used. The proposed method consists of the two following steps:

- Step 1: Bilateral filtering in the patch-space

- Step 2: Projection back onto the image space by using the division of two components of the filtered values (equation (5))

In the first step, the filtered values $\widehat{\boldsymbol{U}}(\boldsymbol{p})$ are given by:

$$
\widehat{U}_{i}(\boldsymbol{p})=\frac{\sum_{\boldsymbol{q} \in \Psi} w_{i}(\boldsymbol{p}, \boldsymbol{q}) V_{i}(\boldsymbol{q})}{\sum_{\boldsymbol{q} \in \Psi} w_{i}(\boldsymbol{p}, \boldsymbol{q})}
$$

where subscript $\mathrm{i}=1,2$, and according to BF's principle the weight $w_{i}(\boldsymbol{p}, \boldsymbol{q})$ is estimated as follows:

$$
\begin{aligned}
& w_{i}(\boldsymbol{p}, \boldsymbol{q}) \\
& =\exp \left(\frac{-\left\|V_{i}(\boldsymbol{p})-V_{i}(\boldsymbol{q})\right\|^{2}}{h^{2}}\right) \exp \left(\frac{-\|\boldsymbol{p}-\boldsymbol{q}\|^{2}}{h_{r}^{2}}\right)
\end{aligned}
$$

where the first term is an intensity proximity measure and the second one stands for a geometric proximity measure. Recall that this second term is the patch similarity measure defined in the image spatial domain. The filtered values $\widehat{\boldsymbol{U}}(\boldsymbol{p})$ are finally projected back onto the image domain $\Omega$ using the division in equation (5). Note that when $h=\infty$ which implies $\exp \left(\frac{-\left\|V_{i}(\boldsymbol{p})-V_{i}(\boldsymbol{q})\right\|^{2}}{h^{2}}\right)=1$ then the proposed method tends to the classical NLM.

\section{EXPERIMENTAL RESUTLS}

The experimental results are carried out on four natural images: Lena, Barbara, Peppers and Fingerprint. The last image is typical of highly textured image whereas the third one contains mostly homogenous regions. The first and second images contain different types of features: texture, sharp edges and smooth regions. These images are perturbed by additive, independent Gaussian noise at two levels of standard deviation: $\sigma=10$ and $\sigma=20$. According to the original work in [5-7], the optimal range parameter $h_{r}$ for NLM is set equal to $\sigma$. In our experience, we test with several values $h=n \sigma$ where $n$ takes a set of values from 2 to 20. Recall that when $n=\infty$, i.e. $h=\infty$ the proposed method behaves like the classical NLM filter. The subspace $\Omega(x, y)$ is defined by small windows $21 \times 21$ around the pixel $(x, y)$. The patch size is equal to $7 \times 7$ which results in patchspace of dimension 49. A comparative evaluation using objective and subjective measures has been performed to demonstrate the advantages of our method over NLM filter. To objectively evaluate the results, we use five fidelity measures as well as a distortion measure, i.e. blurring measure which could be used as an estimate of the smoothing effect. For blurring measure, we use no-reference metric namely BLUR proposed in [17]. Note that higher this value, more blur will result on the image. For fidelity evaluation, beside PSNR, we use also four other metrics namely MAD [18], PSNR ${ }_{W}$ [19], VSNR[20] and VIF[21] which are based on some properties of the human visual system (HVS). Note that while small value of MAD indicates high level of image quality, small value of $\mathrm{PSNR}_{\mathrm{W}}, \mathrm{VSNR}, \mathrm{VIF}$ corresponds to a low level of image quality. A good filtering method must yield higher $\mathrm{PSNR}_{\mathrm{W}}$, VSNR, VIF (or smaller MAD) and smaller BLUR. The results are reported in Fig.3-8 where the abscissa is $n$. The right-most points correspond to $n=\infty$, i.e. NLM method. As can be seen in Fig.3, BLUR values are highest on the right-most part, i.e. NLM's results are oversmoothed. For PSNR measurement, the best result can be found for $n=4$ for all type of images and noise levels. This remark also can be drawn for VIF metric. In the case of PSNR ${ }_{W}$ and MAD, similar results are obtained except for the case of Fingerprint image where the best result is obtained for $n=2$. As can be seen on VNSR curves, the optimal $n$ is found between 2 and 4 . Some conclusions can be drawn here: (i) NLM oversmooths images, (ii) the proposed method outperforms NLM in all case (this is justified by several objective metrics), (iii) the optimal $h$ can be found from $2 \sigma$ to $4 \sigma$.

For subjective evaluation, some images are shown in Fig.9-12 in the case $\sigma=10$. As can be seen in Fig.9, the small details on the hat of Lena are well preserved with the proposed method whereas they are oversmoothed in NLM. It is also easy to see that the homogenous regions in Barbara and Peppers images are oversmoothed in NLM's results while some natural noise is still conserved in our cases. In the case of Fingerprint image, it is little difficult to subjectively judge cause of texture masking effect.

\section{CONCLUSIONS}

A new nonlinear anisotropic filtering method based on BF and a patch-space analysis of the image is proposed. Through this study it has been shown that NLM can be expressed as an isotropic filter in this patch-space. A series of tests has been performed to assess the efficiency of the proposed method. The obtained results demonstrate the efficiency of the proposed filtering approach objectively and subjectively. As a perspective, some improvements will be 
investigated and especially on the computational and complexity sides and the extension to color images.

\section{REFERENCES}

[1] C. Tomasi, R. Manduchi, "Bilateral Filtering for Gray and Color Images" in Proc. ICCV, 1998.

[2] D. Barash, "A Fundamental Relationship between Bilateral Filtering, Adaptive Smoothing, and the Nonlinear Diffusion Equation," Journal of IEEE Transactions on Pattern Analysis and Machine Intelligence, vol. 24, June 2002.

[3] D. Barash, D. Comaniciu, "A common framework for nonlinear diffusion, adaptive smoothing, bilateral filtering and mean shift," Journal of Image and Video Computing, vol. 22, 2004.

[4] M. Elad, "On the origin of the bilateral filter and ways to improve it," IEEE Transactions on Image Processing, vol. 11, pp. 1141-1151, 2002

[5] A. Buades, B. Coll and J. M. Morel, "A review of image denoising algorithms, with a new one," Multiscale Model Simulation Journal, vol. 4, no. 2, pp. 490-530, 2005.

[6] A. Buades, B. Coll and J. M. Morel, "Image and movie denoising by nonlocal means," International Journal of Computer Vision, vol. 76, 2008.

[7] A. Buades, B. Coll and J.M. Morel, "A non-local algorithm for image denoising," IEEE Computer Society Conference on Computer Vision and Pattern Recognition (CVPR'05), vol.2, 2005.

[8] B. Goossens, H.Q. Luong, A. Pizurica, and W. Philips, "An Improved Non-Local Denoising Algorithm", in Proc. International Workshop on Local and Non-Local Approximation in Image Processing, pp. 143-156, 2008.

[9] B. R. C. and M. Vehvilainen, "Fast nonlocal means for image denoising," in Proceedings of SPIE Digital Photography III, vol.6502, no.1, 2007.

[10] W. Souidene, A. Beghdadi, K. Abed-Meraim, "Image denoising in the transformed domain using non local neighborhood”, IEEE - ICASSP, May 15-19, 2006.

[11] D. Tschumperle and L. Brun, "Image Denoising and Registration by PDE's on the Space of Patches," In International Workshop on Local and Non-Local Approximation in Image Processing, 2008.

[12] Ch. Kervrann and J. Boulanger, "Optimal spatial adaptation for patch-based image denoising." IEEE Trans. Image Process., vol. 15, pp. 2866-2878, 2006.

[13] Q.B. Do, M. Luong, and A. Beghdadi, "Combination of closest space and closest structure to ameliorate non-local means method," IEEE Symposium on Computational Intelligence for Multimedia, Signal and Vision Processing, 2011.
[14] C. Barnes, E. Shechtman, D. Goldman, and A. Finkelstein, "The Generalized PatchMatch Correspondence Algorithm," in European Conference on Computer Vision, 2010.

[15] L. Rudin, S. Osher, E. Fatemi, “Nonlinear Total Variation based noise removal algorithms", Physica D 60 259-268,1992.

[16] P. Perona and J. Malik, "Scale-Space and Edge Detection Using Anisotropic Diffusion," IEEE Transactions on Pattern Analysis and Machine Intelligence, vol. 12, 1990.

[17] F. Crete-Roffet, "Estimer, mesurer et corriger les artefacts de compression pour la télévision numérique," thesis in Université Joseph-Fourier - Grenoble I, 2007.

[18] E.C. Larson, D.M. Chandler, "Most apparent distortion: fullreference image quality assessment and the role of strategy," Journal of Electronic Imaging, 19 (1), 2010.

[19] A. Beghdadi, B. Pesquet-Popescu, "A New Image Distortion Measure Based Wavelet Decomposition," In Proc. ISSPA, pp.485488, 2003.

[20] D.M. Chandler, S.S. Hemami, "VSNR: a wavelet-based visual signal-to-noise ratio for natural images," IEEE Trans. Image Process., pp. 2284-98, 2007.

[21] H.R. Sheikh.and A.C. Bovik, "Image information and visual quality," IEEE Transactions on Image Processing, vol.15, no.2,pp. 430- 444, Feb. 2006. 


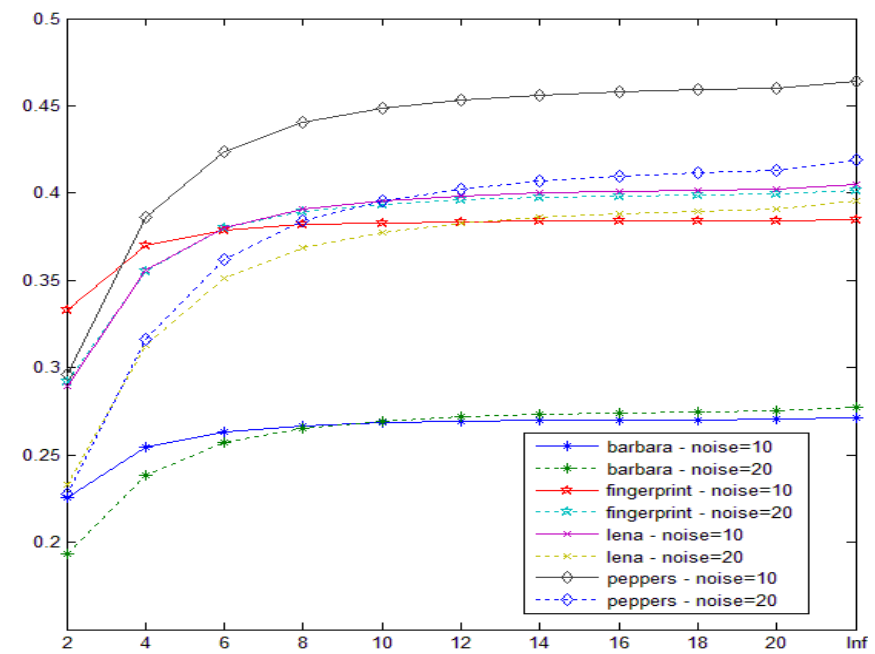

Fig.3: BLUR measurement (the abscissa is $n$ )

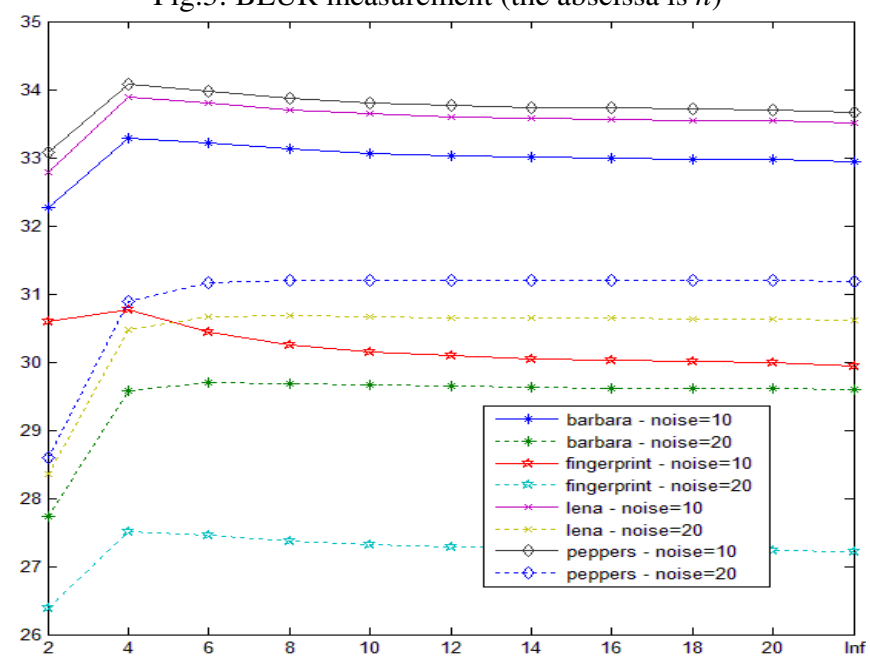

Fig.5: PSNR measurement (the abscissa is $n$ )

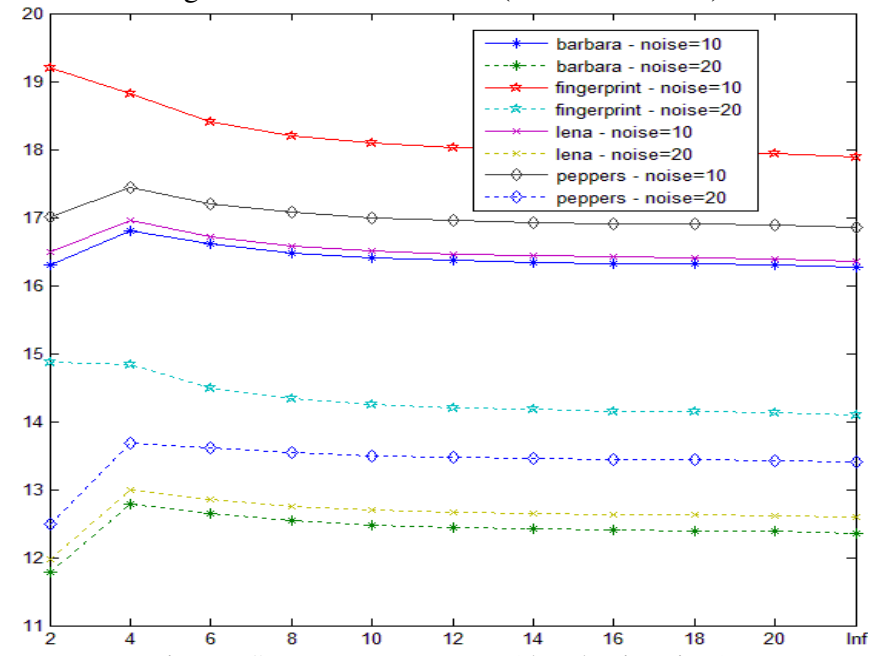

Fig.7: PSNR $_{\mathrm{W}}$ measurement (the abscissa is $n$ )

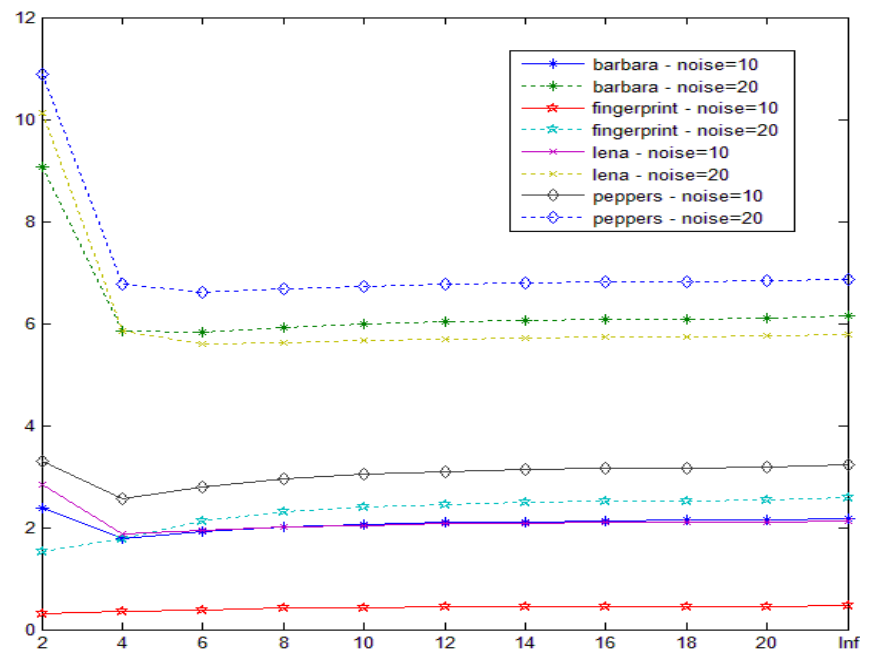

Fig.4: MAD measurement (the abscissa is $n$ )

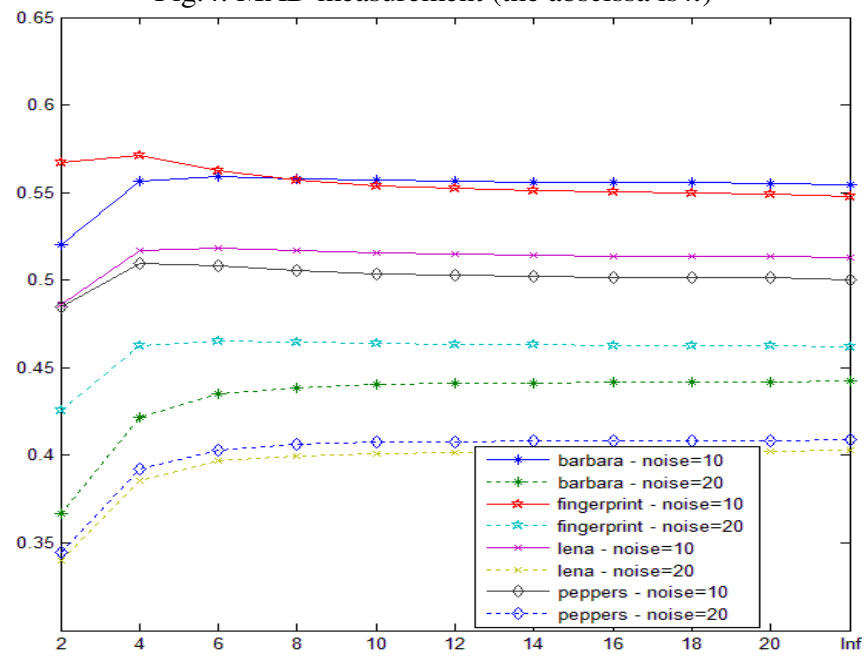

Fig.6: VIF measurement (the abscissa is $n$ )

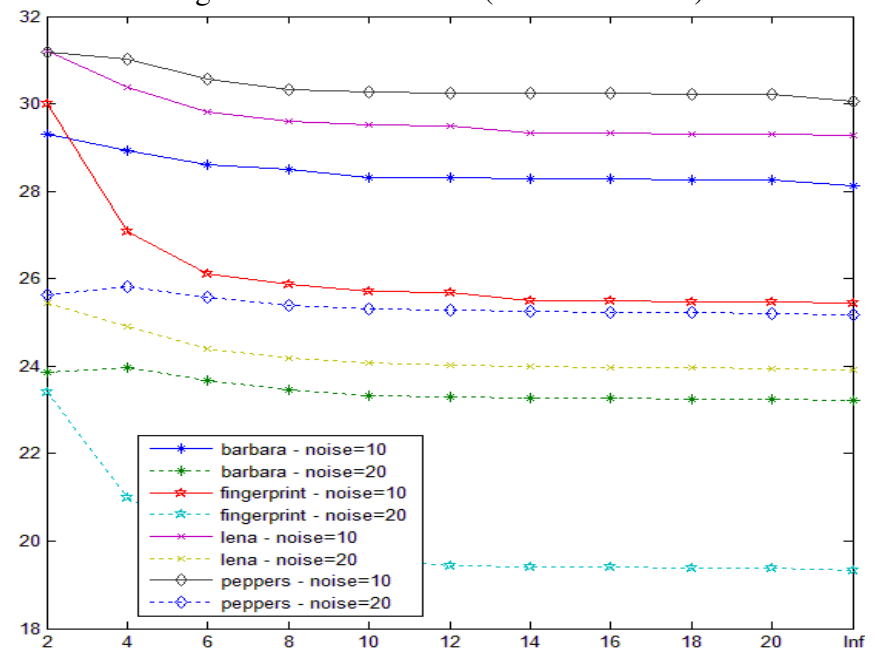

Fig.8: VSNR measurement (the abscissa is $n$ ) 


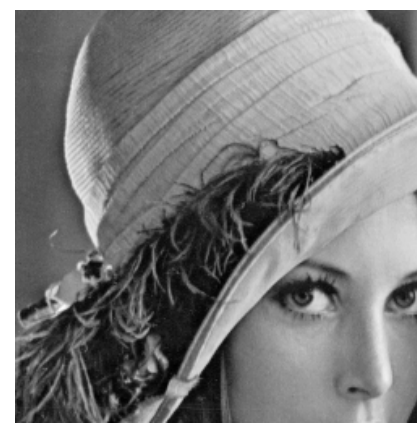

(a)

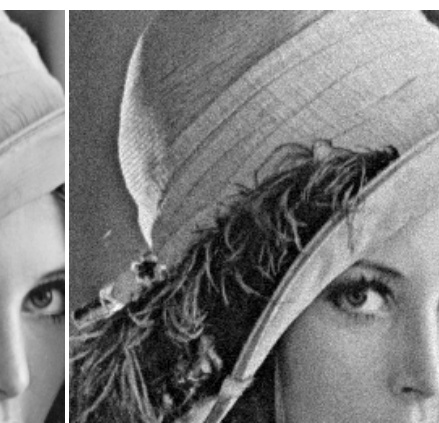

(b)

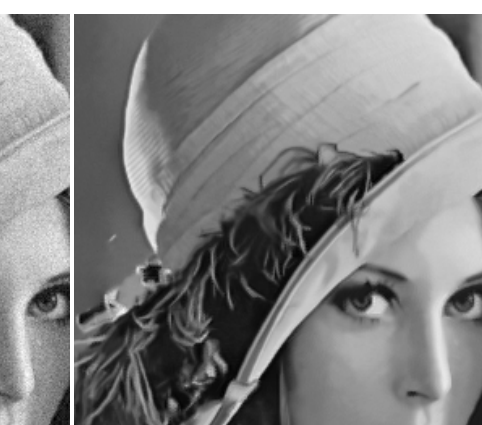

(c)

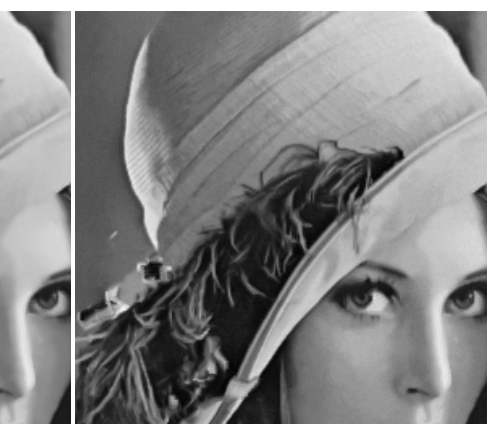

(d)

Fig.9: Lena images (from left to right) the original image, the noisy image, NLM filtering, proposed method

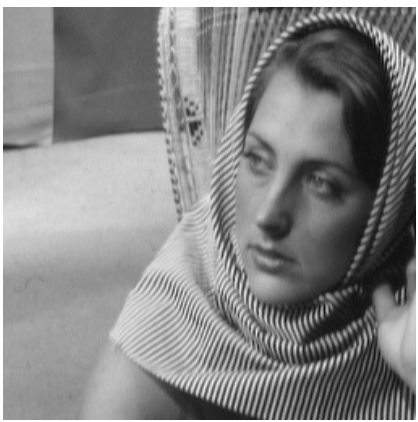

(a)

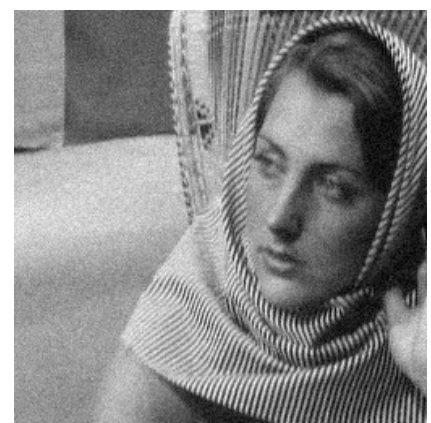

(b)

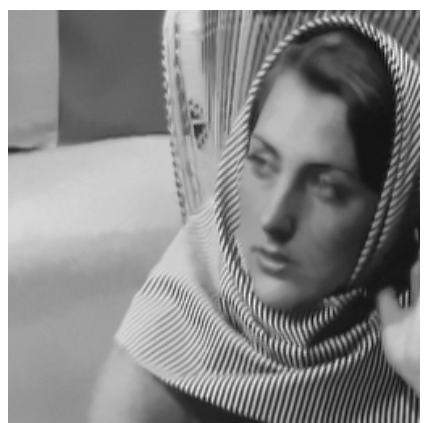

(c)

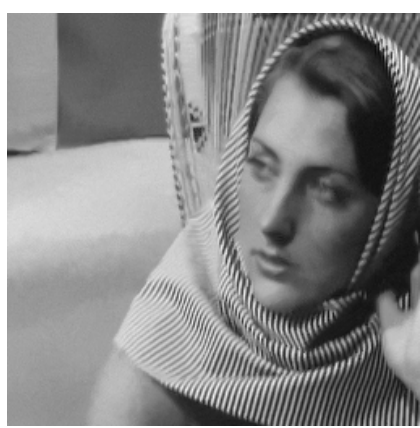

(d)

Fig.10: Barbara images (from left to right) the original image, the noisy image, NLM filtering, proposed method

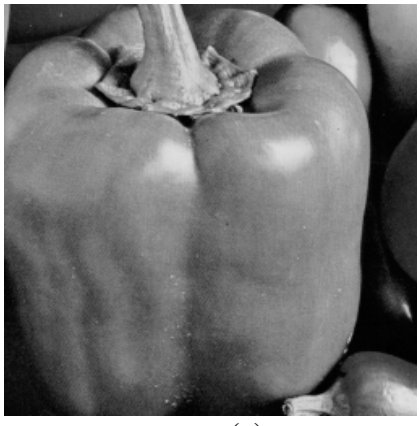

(a)

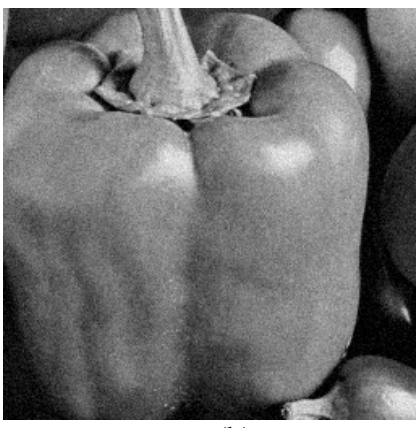

(b)

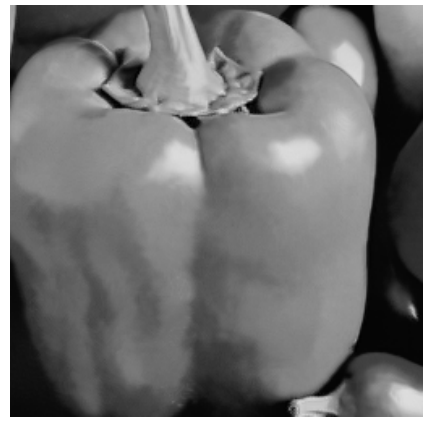

(c)

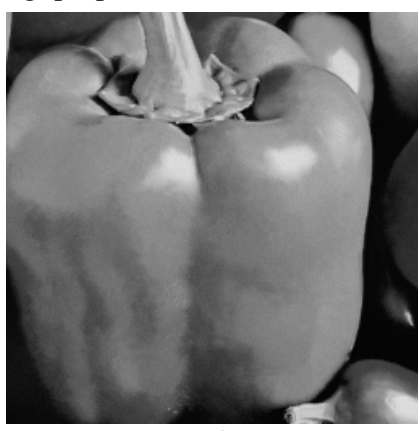

(d)

Fig.11: Peppers images (from left to right) the original image, the noisy image, NLM filtering, proposed method

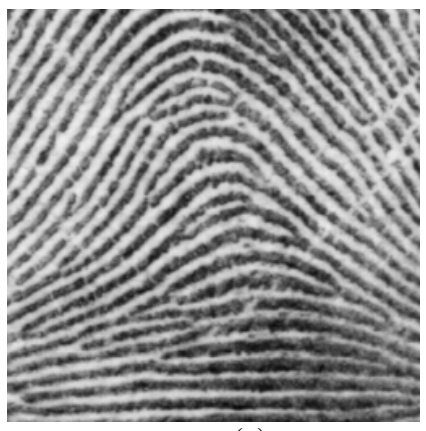

(a)

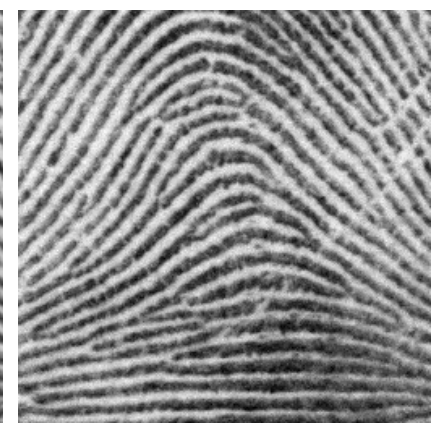

(b)

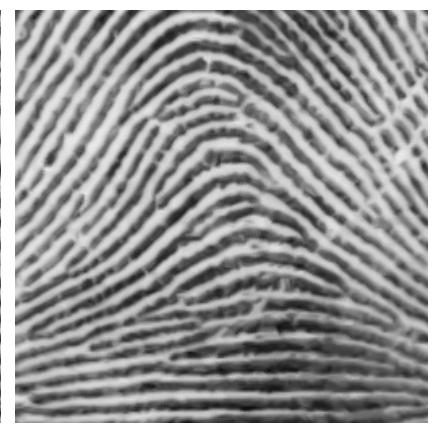

(c)

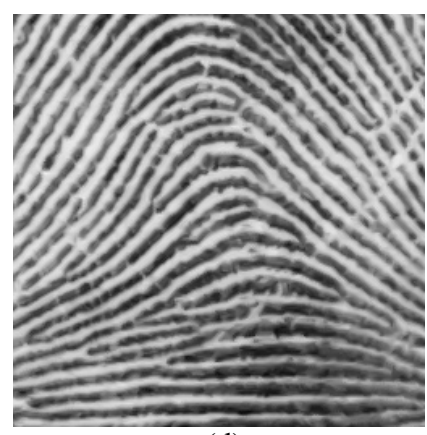

(d)

Fig.12: Fingerprint images (from left to right) the original image, the noisy image, NLM filtering, proposed method 